\title{
Thermally assisted peeling of an elastic strip in adhesion with a substrate via molecular bonds
}

\author{
Jin Qian $^{\text {a }}$, Ji Lin ${ }^{\text {a }}$, Guang-Kui Xu ${ }^{\mathrm{b}}$, Yuan Lin ${ }^{\mathrm{c}, \mathrm{d}, *}$, Huajian Gao ${ }^{\mathrm{e}, *}$ \\ ${ }^{a}$ Department of Engineering Mechanics, Key Laboratory of Soft Machines and Smart Devices of \\ Zhejiang Province, Zhejiang University, Hangzhou, Zhejiang 310027, China \\ ${ }^{b}$ International Center for Applied Mechanics, State Key Laboratory for Strength and Vibration of \\ Mechanical Structures, Xi'an Jiaotong University, Xi'an 710049, China \\ ${ }^{\mathrm{c}}$ Department of Mechanical Engineering, The University of Hong Kong, Hong Kong, China \\ ${ }^{\mathrm{d}}$ HKU-Shenzhen Institute of Research and Innovation (HKU-SIRI), Shenzhen, Guangdong, China \\ ${ }^{\text {e }}$ School of Engineering, Brown University, Providence, Rhode Island 02912, USA
}

* To whom correspondence should be addressed. E-mail: ylin@hku.hk (Y.L.) or huajian_gao@brown.edu (H.G.)

\begin{abstract}
A statistical model is proposed to describe the peeling of an elastic strip in adhesion with a flat substrate via an array of non-covalent molecular bonds. Under an imposed tensile peeling force, the interfacial bonds undergo diffusion-type transition in their bonding state, a process governed by a set of probabilistic equations coupled to the stretching, bending and shearing of the elastic strip. Because of the low characteristic energy scale associated with molecular bonding, thermal excitations are found to play an important role in assisting the escape of individual molecular bonds from their
\end{abstract}


bonding energy well, leading to propagation of the peeling front well below the threshold peel-off force predicted by the classical theories. Our study establishes a link between the deformation of the strip and the spatiotemporal evolution of interfacial bonds, and delineates how factors like the peeling force, bending rigidity of the strip and binding energy of bonds influence the resultant peeling velocity and dimensions of the process zone. In terms of the apparent adhesion strength and dissipated energy, the bond-mediated interface is found to resist peeling in a strongly rate-dependent manner.

Keywords: peeling; molecular bond; thermal fluctuation; cohesive law; rate dependence; energy dissipation

\section{Introduction}

The peel test of a strip from a substrate has been widely used to explore the interfacial properties of a variety of engineering and biological systems. For example, in transferring micro- or nano-scale materials from one substrate to another with an elastomeric stamp, the distinct strength of materialand substrate-stamp interfaces serves as a critical control, and therefore needs to be carefully characterized under the standard peeling configuration (Chen et al., 2013). In addition, both experimental and computational studies on metal-elastomer interfaces have shown that their response to peeling is strongly rate-dependent due to the dissipation of fibrillar microstructures formed in the so-called process zone (Vossen et al., 2014; Neggers et al., 2015). The factors such as material heterogeneity (Xia et al., 2013), adhesive pattern (Xia et al., 2015) and surface corrugation (Jin and Guo, 2013; Zhang et al., 2014; Peng and Chen, 2015) on the enforced separation of thin films from 
substrates have also been extensively examined. The strip-substrate peeling problem was also used as a model system to investigate the ability of geckos to cling to surfaces as well as the role of pre-tension in geckos' feet that gives rise to reversible adhesion (Pesika et al., 2007; Chen et al., 2009; Peng and Chen, 2012).

On the theoretical side, substantial attention has been paid to modeling the large deformation of materials (Molinari and Ravichandran, 2008; Begley et al., 2013) and the occurrence of frictional sliding during the peeling process. Lu et al. (2007) presented a model to examine the role of shear-induced interfacial slippage in the peel test, while a uniform cohesive traction in the adhered region has been introduced to simulate this phenomenon (Gialamas et al., 2014). Cheng et al. (2012) found that interfacial sliding leads to a linear distribution of pre-tension in a thin film being peeled off from a substrate. A recent study has also considered the combined effects of large deformation and interfacial friction/sliding in peeling of an elastic tape from a substrate (Begley et al., 2013). A common viewpoint from all these investigations is that there exists a critical level of peel force for continuous surface detachment to take place, following Kendall $(1971,1975)$. Below the critical peel force, detachment would never occur.

Here we focus on a particular type of peeling mediated by specific molecular bonds, commonly referred to as receptor-ligand bonds (Alberts et al., 2002). Molecular bonds have been found responsible for the interaction between cells and extracellular matrices and dominate many adhesion-mediated cellular processes (Gao et al., 2011; Xu et al., 2014), such as wound healing or tumor development and growth (Roca-Cusachs et al., 2012; Kumar and Weaver, 2009). A thermodynamic framework for describing the behaviors of bond-mediated interfaces was first established by Bell (1978). The processes of adhesion/de-adhesion of non-responsive cells to/from 
substrates were subsequently considered within the context of standard peel tests, accounting for the complications of bio-interfaces and geometries involved (Evans 1985a; 1985b; Dembo et al., 1988; Freund and Lin, 2004). For example, Evans (1985a, 1985b) developed a detailed peeling model of membrane-membrane adhesion with continuous or discrete distribution of cross-bridges between two surfaces; Dembo et al. (1988) studied this problem by coupling the deformation of elastic membrane with rate processes of bond formation and breakage. Quantities of key interest in this kind of studies are the critical peeling force required to separate the membrane-substrate interfaces, and the propagation speed of the peeling front as a function of the applied load.

The essential feature that distinguishes the present study from the conventional peeling problems is the specific lock-and-key mechanism of interfacial bonding, which can transit stochastically between a closed state and an open state. This stochastic nature is rooted in the relatively low characteristic energy scale associated with molecular bonding, which has a typical value of 10-25 $k_{\mathrm{B}} T_{\mathrm{m}}$ (Leckband and Israelachvili, 2001), $k_{\mathrm{B}}$ being the Boltzmann constant and $T_{\mathrm{m}}$ the absolute temperature. Because this energy is comparable to thermal energy, a molecular bond can undergo reversible transitions between the closed and open states in a thermally assisted manner, with or without other driving forces. In other words, any closed bonds will eventually break if sufficiently long time is allowed, and any broken bonds can reform if proximity is maintained (Qian et al., 2013; $\mathrm{Ju}$ et al., 2015). At single-bond level, such bi-directional binding and unbinding are commonly characterized by measuring their association and dissociation kinetic rates, which were shown to be tightly regulated by physical factors (Evans and Ritchie, 1997; Rief et al., 1997; Merkel et al., 1999) as well as biochemical signals that are still being elucidated. For clusters of multiple bonds, augment in cluster size was shown to enhance the strength and stability of the ensemble (Erdmann and 
Schwarz, 2004a; 2004b), but this trend is limited because large clusters can be weakened by stress concentration (Qian et al., 2008; 2009). The collective behavior of such molecular bonds is crucial for our understanding of various adhesion and de-adhesion phenomena between cells and extracellular matrices (Freund, 2014; Wei, 2014; Jiang et al., 2015).

The present study aims to develop a theoretical model to describe the peeling progression of an elastic strip adhered to a substrate via an array of receptor-ligand bonds. Interfacial bonding and debonding are described by the spatiotemporal evolution of adhesive states of individual bonds, subjected to the imposed surface separation as well as thermal excitations in a strongly rate-dependent manner. Our statistical approach shows that the peeling can progress diffusively at any value of the applied load, in contrast to the commonly held viewpoint from most existing models that a critical level of peel force must be reached before debonding between two surfaces can possibly occur.

\section{Model}

\subsection{Governing equations of strip deformation under peeling}




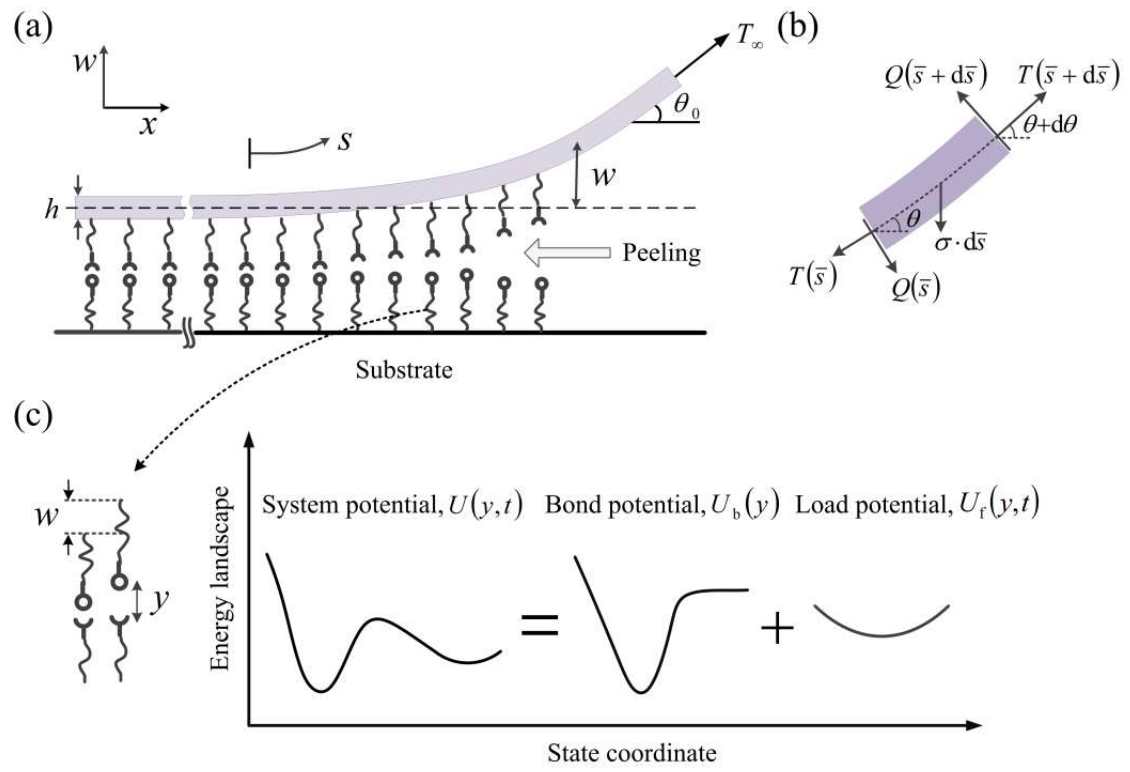

Fig. 1. Schematic of the peeling model under investigation. (a) A strip being peeled off from a substrate by an inclined tensile force $T_{\infty}$ on its right end with molecular bonds uniformly distributed along the strip-substrate interface. An arbitrary point in the strip can be identified by its arch length coordinate $s$ whereas its horizon position is described by $x$. (b) The equilibrium of a strip segment in two orthogonal directions, one tangent and the other normal to the strip. (c) The binding energy landscape (summation of bond potential and load potential) of a molecular bond at the peeling interface. The state of interfacial bonding can be described by the evolution of probability distribution along the state coordinate.

Consider the standard configuration of a peel test in which a flexible strip is peeled off from a flat rigid substrate by the application of a tensile force $T_{\infty}$ at one end, as depicted in Fig. 1(a). The peel angle $\theta_{0}$ is fixed as the peeling front propagates at a steady-state velocity $v$. The quantitative relation between $T_{\infty}$ and $v$ is of central interest in the present problem. Along the peeling interface, the opposing surfaces are brought together via non-covalent molecular bonds, which can assume different bonding states. In the following, we will first present the governing equations of the deformation of the elastic strip under peeling, followed by a statistical description in predicting the spatiotemporal evolution of the interfacial bonding state.

The position along the strip profile is identified by its arch length coordinate $s$ while the shape of the strip at time $t$ is described by its deflection $w(s, t)$ in the direction perpendicular to the 
horizontal $x$-axis (referring to Fig. 1). If the peeling front propagates with a steady-state velocity $v$, the coordinate in the frame translating with the front is simply $\bar{s}=s+v t$. On the basis of the steady-state, all physical quantities become invariant with respect to time in the moving frame, as analyzed in the work of Evans (1985a, 1985b) and Dembo et al. (1988). As such, the strip deflection is invariant with respect to time in the moving frame, and becomes a single variable function only depending on the moving coordinate $\bar{s}$, i.e. $w(\bar{s})$. If the adhesive traction provided by the molecular bonds within a unit area of the strip-substrate interface is $\sigma$, in the direction of $w$, the equilibrium of the strip in two orthogonal directions, one tangent and the other normal to the strip (Fig. 1(b)), requires that

$$
\begin{aligned}
& \frac{\mathrm{d} T}{\mathrm{~d} \bar{s}}-Q \frac{\mathrm{d} \theta}{\mathrm{d} \bar{s}}-\sigma \frac{\mathrm{d} w}{\mathrm{~d} \bar{s}}=0 \\
& \frac{\mathrm{d} Q}{\mathrm{~d} \bar{s}}+T \frac{\mathrm{d} \theta}{\mathrm{d} \bar{s}}-\sigma \frac{\mathrm{d} x}{\mathrm{~d} \bar{s}}=0
\end{aligned}
$$

where $T$ and $Q$ are the tension and shear force within the strip, and $\theta$ stands for the tangent angle of the strip profile. Note that the local curvature $K$ of the deformed strip is $\mathrm{d} \theta / \mathrm{d} \bar{s}$, and the shear force can be expressed in terms of $K$ and the bending modulus of the strip $B$ as $Q=-B \mathrm{~d} K / \mathrm{d} \bar{s}$. With these observations, Eqs. (1) and (2) become

$$
\begin{aligned}
& \frac{\mathrm{d} T}{\mathrm{~d} \bar{s}}+B K \frac{\mathrm{d} K}{\mathrm{~d} \bar{s}}-\sigma \frac{\mathrm{d} w}{\mathrm{~d} \bar{s}}=0, \\
& B \frac{\mathrm{d}^{2} K}{\mathrm{~d} \bar{s}^{2}}-T K+\sigma \frac{\mathrm{d} x}{\mathrm{~d} \bar{s}}=0
\end{aligned}
$$

The curvature $K$ can also be related to the strip deflection as $K=\left(\mathrm{d}^{2} w / \mathrm{d} \bar{s}^{2}\right) /(\mathrm{d} x / \mathrm{d} \bar{s})$. The boundary conditions are

$$
\begin{gathered}
T(\bar{s}) \rightarrow T_{\infty}, \quad \mathrm{d} w(\bar{s}) / \mathrm{d} \bar{s} \rightarrow \sin \theta_{0}, \text { as } \bar{s} \rightarrow+\infty \\
w(\bar{s}) \rightarrow 0, \quad \mathrm{~d} w(\bar{s}) / \mathrm{d} \bar{s} \rightarrow 0, \text { as } \bar{s} \rightarrow-\infty
\end{gathered}
$$


where $T_{\infty}$ and $\theta_{0}$ denote the peeling force and angle, respectively. The conditions in Eq. (6) ensure that the strip deflection and its derivative vanish far from the process zone of peeling. At this point, the remaining question is to calculate the interfacial traction $\sigma$ contributed by the molecular bonds.

\subsection{Statistical descriptions of interfacial bonding and debonding}

We postulate that all the interfacial bonds are nominally identical with the same mechanical and kinetic properties, but can assume totally different bonding states under peeling because of their stochastic nature. Let us focus on a strip segment, between $\bar{s}$ and $\bar{s}+\mathrm{d} \bar{s}$, that is in adhesive binding with the substrate via a number of such randomly distributed bonds. Far from the process zone of peeling, the strip is separated from the substrate by a distance $l_{\mathrm{b}}$, which is taken to be the undeformed bond length plus the equilibrium distance between the binding entities of receptor and ligand molecules. At a given coordinate $\bar{s}$, the bonds collectively generate a resisting force against the induced surface separation $w(t)$. Microscopically, each molecular bond under stretching behaves like a Hookean spring whose bonding-debonding transition can be described as the spatiotemporal evolution of bond state distribution between an energy minimum and a transitional activation barrier within a prescribed energy landscape (Freund, 2009a; Lin et al., 2014).

We proceed by assuming that the inherent interaction of individual bonds is characterized by a one dimensional potential energy $U_{\mathrm{b}}(y)$, where $y$ is the state coordinate defined as the separation between the interacting molecule pairs relative to their equilibrium configuration (identified as $y=0$ in the present setting). Bond dissociation occurs with increased $y$ along the pulling direction, while at the same time, broken bonds may reform because of the returning flux that decreases $y$. For the purpose of quantitative demonstration, we adopt the same energy well profile adopted by 
Freund (2009a), namely,

$$
U_{\mathrm{b}}(y)=\left\{\begin{array}{cc}
C_{\mathrm{b}} k_{\mathrm{B}} T_{\mathrm{m}}\left(2 y^{2} / a^{2}-1\right), & -\infty<y / a \leq 0.5 \\
-2 C_{\mathrm{b}} k_{\mathrm{B}} T_{\mathrm{m}}(y / a-1)^{2}, & 0.5<y / a<1, \\
0, & 1 \leq y / a \leq+\infty
\end{array},\right.
$$

where $C_{\mathrm{b}}$ is a dimensionless number describing the depth of the energy well in units of thermal energy $k_{\mathrm{B}} T_{\mathrm{m}}$, and $a$ represents the half-width of the well that is usually on the order of $1 \mathrm{~nm}$, depending on the bond type (Bell, 1978; Evans and Ritchie, 1997).

The influence of external pulling can be quantified by adding an extra term of potential energy (denoted as $U_{\mathrm{f}}$ ) with the form:

$$
U_{\mathrm{f}}(y, t)=k(w(t)-y)^{2} / 2
$$

where $k$ represents the spring constant of the bonds while $w(t)$ is the strip deflection at spatial coordinate $\bar{s}$. Now, the bond evolution and strip deformation are coupled. Once $U_{\mathrm{b}}$ and $U_{\mathrm{f}}$ are known, the collective response of interfacial bonds to pulling can be described by how their internal states evolve temporally along the combined energy landscape:

$$
U(y, t)=U_{\mathrm{b}}(y)+U_{\mathrm{f}}(y, t) .
$$

Despite being heavily influenced by the separation profile $w(t)$, the actual profile of $U(y, t)$ typically exhibits an energy minimum separated at a certain distance from an energy barrier (e.g., Fig. 1(c)). The internal state of any bond, as represented by its coordinate $y$, will evolve over time within this energy profile akin to a random walk (Kramers, 1940). Statistically, the distribution of non-interacting states among an ensemble of indistinguishable bonds can be described by a probability density function, denoted by $\rho(y, t)$. Local conservation requires that the rate of accumulation or loss in $\rho(y, t)$ must be attributed to the local divergence of leaving and entering fluxes, namely, 


$$
\frac{\partial \rho(y, t)}{\partial t}=-\frac{\partial j(y, t)}{\partial y}
$$

where $j(y, t)$ is the flux of bond state in the $y$ direction at time $t$. With the constitutive assumption that the flux of state is linearly proportional to the local gradient of associated chemical potential, Freund (2009a) showed that $j(y, t)$ can be related to the bond state distribution $\rho(y, t)$ and the combined energy landscape $U(y, t)$ through

$$
j(y, t)=-D\left(\frac{\partial \rho(y, t)}{\partial y}+\frac{\rho(y, t)}{k_{\mathrm{B}} T_{\mathrm{m}}} \frac{\partial U(y, t)}{\partial y}\right),
$$

where $D$ is the diffusivity of bond state.

Choosing $a$ and $a^{2} / D$ as the basic length and time scales in the problem, we combine Eqs. (10) and (11) into the normalized Smoluchowski partial differential equation that governs the spatiotemporal evolution of bond state distribution:

$$
\frac{\partial \hat{\rho}(Y, \tau)}{\partial \tau}=\frac{\partial}{\partial Y}\left(\frac{\partial \hat{\rho}(Y, \tau)}{\partial Y}+\hat{\rho}(Y, \tau) \frac{\partial \hat{U}(Y, \tau)}{\partial Y}\right),
$$

where the normalization scheme is chosen as $Y=y / a, \tau=t D / a^{2}, \hat{\rho}=\rho a$ and $\hat{U}=U / k_{\mathrm{B}} T_{\mathrm{m}}$. Equation (12) is subjected to boundary conditions $\hat{\rho}(-\infty, \tau)=\hat{\rho}(+\infty, \tau)=0$, indicating that the density of bond state should vanish far from the energy well. The initial condition is taken to be $\hat{\rho}(Y, 0)=\delta(Y), \delta(Y)$ being the Dirac delta function, i.e. all bonds are initially intact with internal state of $Y=0$.

Now let us focus on an arbitrarily selected position along the interface, and investigate the time-varying behavior of bond traction at this position. Statistically, the resultant force per unit area between the opposing surfaces, corresponding to the cohesive stress at the interface, is expressed as

$$
\sigma(\tau)=\Omega k a \int_{-\infty}^{+\infty} \hat{\rho}(Y, \tau)(W(\tau)-Y) \mathrm{d} Y
$$

where $\Omega$ is the areal site density of bonds, $k a$ serves as a $\mathrm{pN}$-level force scale, and $W$ is the 
strip deflection normalized by $a$. The expression in Eq. (13) can be understood as follows: at time $\tau, \quad \hat{\rho}(Y, \tau) \mathrm{d} Y$ gives the probability of bonds in state $Y$. These bonds are elongated by $a(W(\tau)-Y)$, and each of them provides a force $k a(W(\tau)-Y), k$ being the bond stiffness. Within a unit area, there are $\Omega \hat{\rho}(Y, \tau) \mathrm{d} Y$ bonds in state $Y$, and integrating over all the possible states gives rise to the cohesive stress in Eq. (13). Substitution of Eq. (13) into Eqs. (3) and (4) closes the mathematical descriptions of the problem.

As the molecular bonds are separated as the strip is peeled away from the substrate, the time trajectory of strip deflection $W$ plays an important role in governing the evolution of bond traction $\sigma(\tau)$. When the strip is peeled off with a normalized steady-state velocity of $V=a v / D$, different locations at the interface are actually temporally correlated. To see this, let us focus on two representative points separated by a distance $\Delta X$, referring to $P_{1}$ and $P_{2}$ in Fig. 2; $P_{1}$ will experience the same loading history as $P_{2}$ when the peeling front propagates by $\Delta X$. In other words, the temporal evolution of bonding status at $P_{1}$ is identical to that at $P_{2}$, with a time lag of $\Delta \tau=\Delta X / V$. Similarly, the full history of bond traction at one single point can also be used to recover the spatial distribution of $\sigma$ along the entire interface.

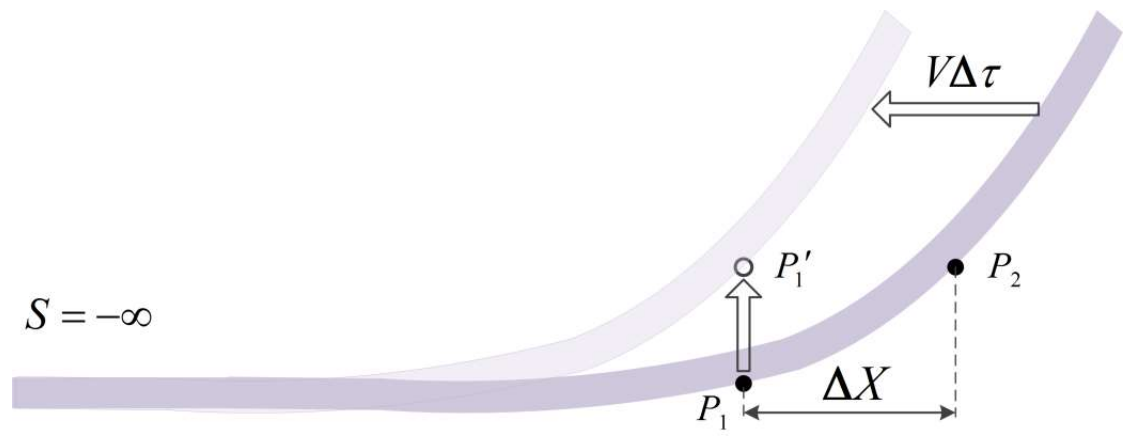

Fig. 2. Similarity of loading history at different points as the peeling front propagates. 


\section{Results and discussion}

\subsection{Numerical solution scheme}

There are four equations (i.e., Eqs (3), (4), (12) and (13)) that govern the bond-mediated peel process. We proceed by normalizing the problem using the following scheme:

$$
\bar{T}=\frac{T}{\Omega k a^{2}}, \quad \bar{\sigma}=\frac{\sigma}{\Omega k a}, W=\frac{w}{a}, X=\frac{x}{a}, \quad S=\frac{\bar{s}}{a}, \bar{K}=K a, \quad \alpha=\frac{\Omega k a^{4}}{B}
$$

The equilibrium conditions in this case become

$$
\begin{aligned}
& \alpha \frac{\mathrm{d} \bar{T}}{\mathrm{~d} S}+\bar{K} \frac{\mathrm{d} \bar{K}}{\mathrm{~d} S}-\alpha \bar{\sigma} \frac{\mathrm{d} W}{\mathrm{~d} S}=0 \\
& \frac{\mathrm{d}^{2} \bar{K}}{\mathrm{~d} S^{2}}-\alpha \overline{T K}+\alpha \bar{\sigma} \frac{\mathrm{d} X}{\mathrm{~d} S}=0
\end{aligned}
$$

while, as pointed out before, the spatiotemporal evolutions of the interfacial traction and bonding state are governed by

$$
\begin{gathered}
\bar{\sigma}(\tau)=\int_{-\infty}^{+\infty} \hat{\rho}(Y, \tau)(W(\tau)-Y) \mathrm{d} Y \\
\frac{\partial \hat{\rho}(Y, \tau)}{\partial \tau}=\frac{\partial}{\partial Y}\left(\frac{\partial \hat{\rho}(Y, \tau)}{\partial Y}+\hat{\rho}(Y, \tau) \frac{\partial \hat{U}(Y, \tau)}{\partial Y}\right) .
\end{gathered}
$$

There are four independent unknown variables here, i.e., $W, \bar{T}, \bar{\sigma}, \hat{\rho}$, and all the other quantities can be expressed in terms of them. In particular, $\mathrm{d} X / \mathrm{d} S=\sqrt{1-(\mathrm{d} W / \mathrm{d} S)^{2}}$, $\bar{K}=\left(\mathrm{d}^{2} W / \mathrm{d} S^{2}\right) /(\mathrm{d} X / \mathrm{d} S)$. At the extremity of the strip, the following asymptotic conditions should be satisfied:

$$
\begin{gathered}
\bar{T}(S) \rightarrow \bar{T}_{\infty}, \mathrm{d} W(S) / \mathrm{d} S \rightarrow \sin \theta_{0}, \text { as } S \rightarrow+\infty, \\
W(S) \rightarrow 0, \mathrm{~d} W(S) / \mathrm{d} S \rightarrow 0, \text { as } S \rightarrow-\infty
\end{gathered}
$$

For the bonding status at the interface, the boundary and initial conditions are

$$
\hat{\rho}( \pm \infty, \tau)=0, \quad \hat{\rho}(Y, 0)=\delta(0)
$$


Note that the strip deflection and its derivative should approach zero as $S \rightarrow-\infty$, implying that

$$
\mathrm{d} S=\mathrm{d} X, \bar{K}=\mathrm{d}^{2} W / \mathrm{d} S^{2} .
$$

Since the interfacial bonds are assumed to provide traction in the direction perpendicular to the substrate surface, the global force equilibrium in the horizontal direction requires that

$$
\bar{T}(-\infty) \rightarrow \bar{T}_{\infty} \cos \theta_{0}
$$

The asymptotic expression of bond traction is therefore $\bar{\sigma}(-\infty)=W(-\infty)$ under the present normalization scheme. Consequently, Eq. (15) is satisfied automatically as $S \rightarrow-\infty$, and Eq. (16) becomes

$$
\frac{\mathrm{d}^{4} W}{\mathrm{~d} S^{4}}-\alpha \bar{T}_{\infty} \cos \theta \frac{\mathrm{d}^{2} W}{\mathrm{~d} S^{2}}+\alpha W=0
$$

Now let us estimate the parameter $\alpha=\Omega \mathrm{ka}^{4} / B$ in this equation. The typical value of bond density $\Omega$ is in the range of $100-1000 \mu \mathrm{m}^{-2}$ since the size of single adhesion molecules (like integrin) is about $10 \mathrm{~nm}$ (Arnold et al., 2004). The bending modulus of cell membrane $B$ varies between 35 and $300 k_{\mathrm{B}} T_{\mathrm{m}}$, depending on the extent of cortex (Boal, 2002; Freund, 2009b). The half-width of bond energy well $a$ falls into nanometer range and the stiffness $k$ of individual bonds is around 1-5 pN/nm (Chen et al., 2012). For possible combinations of the parameters within the range above, $\alpha$ usually has a small value below $10^{-2}$. As such, from Eq. (24), the deflection of the strip should take the asymptotic form as

$$
W(S)=e^{\lambda_{1} S}\left[C_{1} \cos \left(\lambda_{2} S\right)+C_{2} \sin \left(\lambda_{2} S\right)\right]
$$

as $S \rightarrow-\infty$, where $\lambda_{1}=\sqrt{\sqrt{\alpha / 4}+\alpha \bar{T}_{\infty} \cos \theta_{0} / 4}, \lambda_{2}=\sqrt{\sqrt{\alpha / 4}-\alpha \bar{T}_{\infty} \cos \theta_{0} / 4}$ and $C_{1}, C_{2}$ are two constants to be determined.

On the basis of these asymptotic results at the left boundary, a shooting method is developed as we can get enough initial values of the variables in Eqs. (15)-(18) (Lin et al., 2010). Because of the 
$\mathrm{d}^{2} \bar{K} / \mathrm{d} S^{2}$ term, Eqs. (15) and (16) are actually $4^{\text {th }}$-order differential equations of $W, \bar{T}, \bar{\sigma}$ with respect to $S$, which can be discretized with the standard finite difference method. Starting from the left boundary $(S \rightarrow-\infty$ ), which is also set as $X=0$ without loss of generality, the strip deflection at this point is supposed to be $W(X=0)=W_{0}$. With a guessed value of $C_{1}, C_{2}$ can be determined from Eq. (25), which in turn provides sufficient initial values of strip deflection $\left(W_{0}, W_{1}, W_{2}, W_{3}\right)$ for the leading coordinate grids $S_{0}, S_{1}, S_{2}, S_{3}$. In addition, the nodal values of tension within the strip and bond traction, $\bar{T}_{0}, \bar{T}_{1}, \bar{T}_{2}$ and $\bar{\sigma}_{0}, \bar{\sigma}_{1}, \bar{\sigma}_{2}$, can be specified according to $\bar{T}(-\infty) \rightarrow \bar{T}_{\infty} \cos \theta_{0}$ and $\bar{\sigma}(-\infty)=W(-\infty)$ at the left boundary. These initial values allow us to apply the $4^{\text {th }}$-order central difference scheme to Eqs. (15) and (16), resulting in the deflection and tension of the strip at the next point $\left(W_{4}\right.$ and $\left.\bar{T}_{3}\right)$.

The other unknown quantity is the peeling velocity $V$, which is assigned an arbitrary initial value. For the bonds at point $S_{3}$, the loading history on bonds is given by $W_{0}, W_{1}, W_{2}, W_{3}$ that are separated by an incremental time $\Delta X / V$, and the partial differential equation of bond state $\hat{\rho}(Y, \tau)$ can be solved from Eq. (18), which leads to $\bar{\sigma}_{3}$ from Eq. (17).

Repeating the aforementioned procedure point by point, we will eventually obtain the plateau values of the tension and slope profile of the strip, which are compared to the boundary conditions at the peeling end (Eq. (19)). It is easy to imagine that the bond traction will reduce to zero as the strip deflection exceeds a certain value. However, the numerical values of strip tension and deflection are usually not coincident with the boundary conditions at $S \rightarrow+\infty$ because $W_{0}, C_{1}$ and $V$ are arbitrarily selected at the onset. We ameliorate the values of $W_{0}, C_{1}$ and $V$ with Newton's method and seek the final solutions that satisfy the boundary conditions at $S \rightarrow+\infty$. 

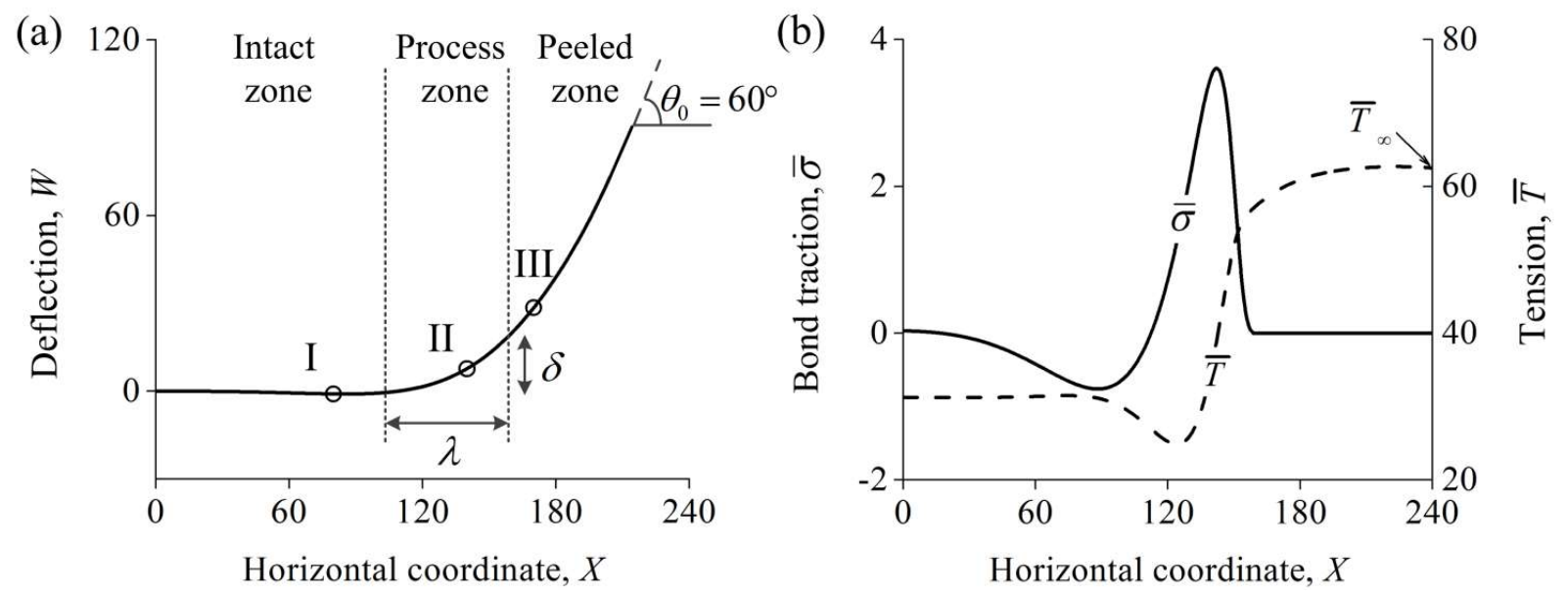

Fig. 3. (a) Deflection profile of the strip in the peeling process. (b) Distributions of bond traction and tension along the strip. The adopted parameters are $\bar{T}_{\infty}=62.5, \theta_{0}=\pi / 3, \alpha=6.25 \times 10^{-6}$ and $C_{\mathrm{b}}=10$.

To present our numerical results, the following bond parameters are chosen:

$$
C_{\mathrm{b}}=10 k_{\mathrm{B}} T_{\mathrm{m}}, k=2 \mathrm{pN} / \mathrm{nm}, a=1 \mathrm{~nm}, \quad D=1 \mathrm{~nm}^{2} / \mathrm{ms} \text {. }
$$

In addition, the values of bond density and strip bending modulus are selected as $\Omega=1000 \mu \mathrm{m}^{-2}$ and $B=80 k_{\mathrm{B}} T_{\mathrm{m}}$, respectively. As such, the normalized parameter $\alpha=\Omega k a^{4} / B$ is calculated to be $6.25 \times 10^{-6}$. The remotely applied peeling force is set to be $\bar{T}_{\infty}=62.5$ with an inclined angle $\theta_{0}=\pi / 3$. These parameters are used throughout our calculations unless stated otherwise. Following the numerical scheme described above, a normalized peeling velocity $V=0.16$ is obtained. The deflection and tension of the strip, as well as the interfacial bond traction, are plotted in Figs. 3(a) and 3(b), respectively. It is noticed that the strip undergoes a little negative deflection before it departs from the substrate surface exponentially. In addition, the magnitude of strip tension equals to $\bar{T}_{\infty} / 2$ at the starting point of $X=0$ because $\theta_{0}=\pi / 3$, but gradually approaches $\bar{T}_{\infty}$ as $X$ increases. In the transition from $S \rightarrow-\infty$ to $S \rightarrow+\infty$, the bond traction first decreases to being compressive, then increases to reach a maximum, beyond which the traction gradually vanishes because of the severe bond rupture when the strip is fully separated from the substrate. 

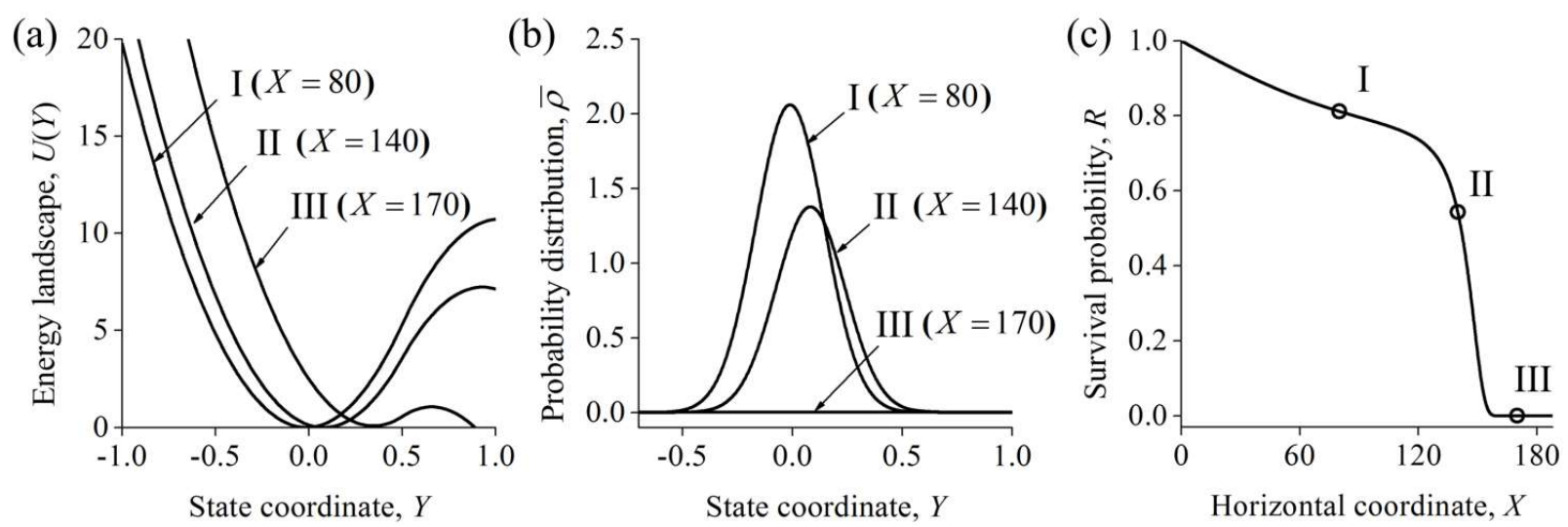

Fig. 4. (a) Landscapes of system energy at indicated positions within the intact zone (I), process zone (II) and peeled zone (III), respectively. (b) Probability distributions of the bond state and (c) survival probability of bonds along the interface. I, II and III represent different positions along the strip appearing in (a).

To understand these results, we record how the energy landscape changes and how the probability distribution of molecular bonds evolves within the intact, process and peeled zone, as shown in Figs. 4(a) and 4(b). In particular, the so-called survival probability of bonds, defined as the probability for them to remain in bonded state, can be evaluated as

$$
R(\tau)=\int_{-\infty}^{Y^{*}} \hat{\rho}(Y, \tau) \mathrm{d} Y
$$

where $Y^{*}$ denotes the location of the energy barrier. This probability decreases monotonously from unity to zero as the strip deflection increases exponentially (Fig. 4(c)). This can be understood by realizing that large deflection of the strip greatly lowers the energy barrier and narrows the gap between the well and the barrier, leading to decreasing or vanishing energy penalty for bonds to escape from their bonding state. 


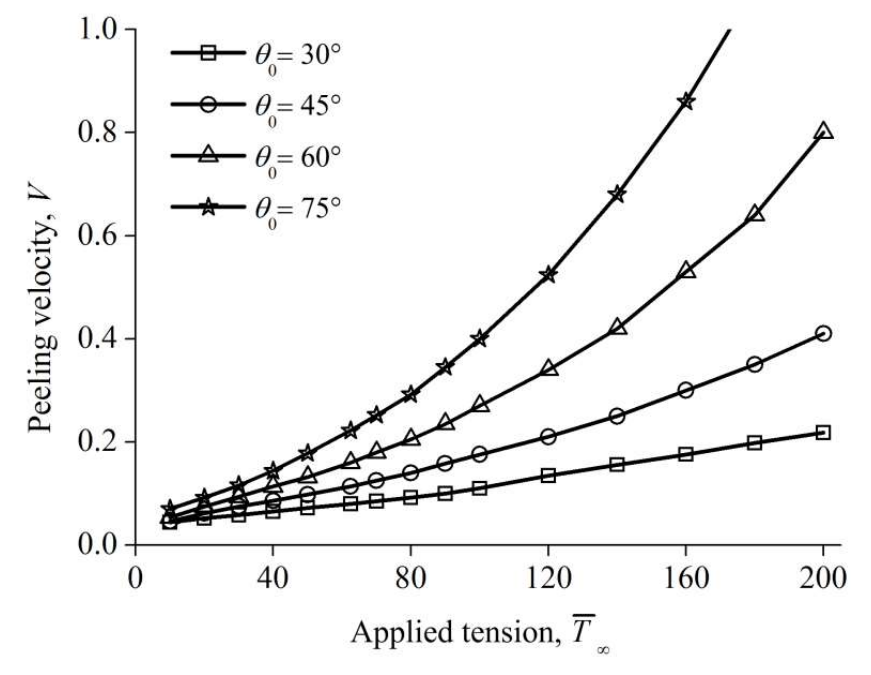

Fig. 5. Peeling velocity as a function of the magnitude of the peeling force applied at various peeling angles.

The remotely applied peeling force serves as the direct driving force that ruptures the strip-substrate interface and propagates the peeling front. Therefore, it is of central interest to examine how this force influences the peeling speed. Figure 5 plots the peeling velocity $V=a v / D$ as a function of the magnitude of the normalized peeling force $\bar{T}_{\infty}$ applied at different peeling angles $\left(\theta_{0}=30^{\circ}, 45^{\circ}, 60^{\circ}, 75^{\circ}\right)$. For each loading angle, the peeling velocity monotonically increases with the applied peeling force. The larger the peeling angle, the faster the peeling speed. These results are consistent with our intuitions. 
(a)

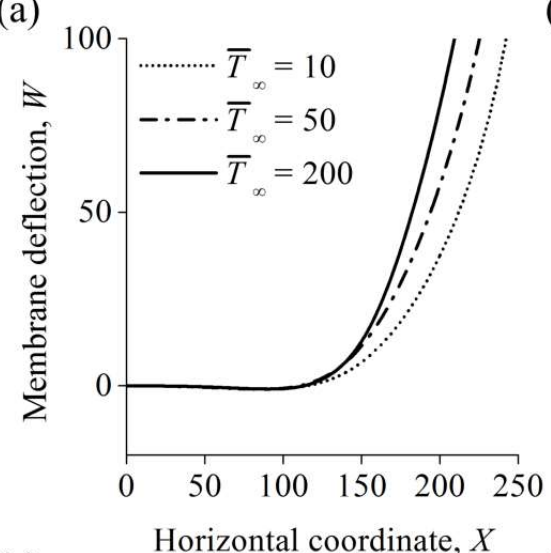

(c)

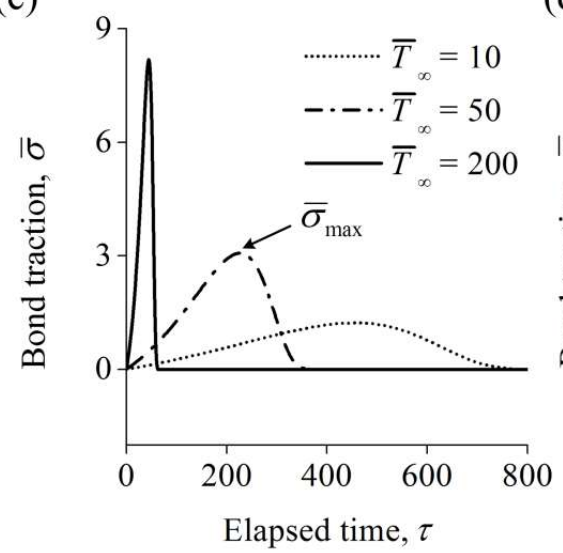

(b)

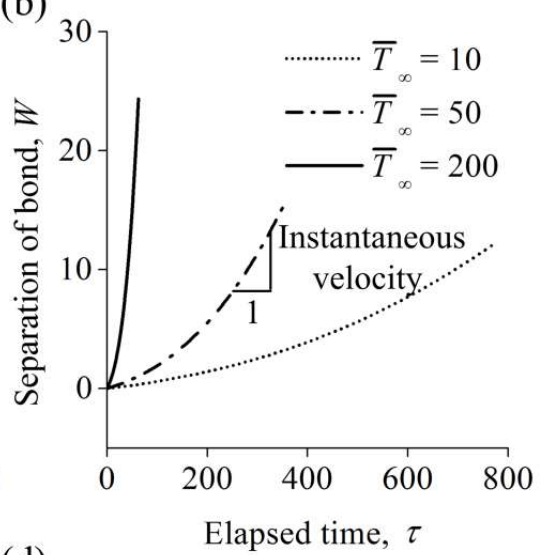

(d)

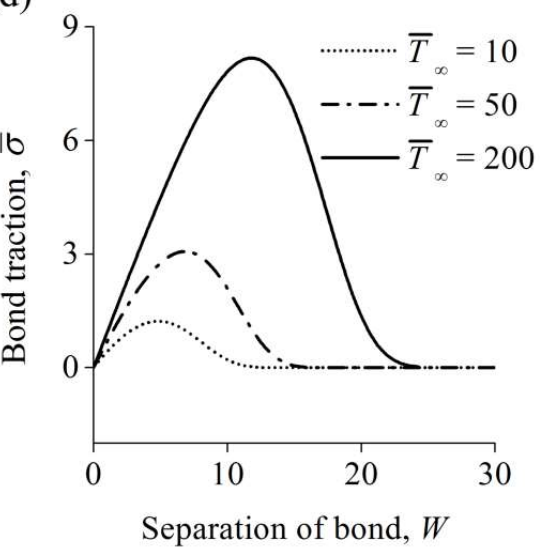

Fig. 6. Rate sensitivity of interfacial response at different levels of the peeling force $\bar{T}_{\infty}$. (a) Strip profiles, and time-varying behaviors of (b) bond separation and (c) bond traction. (d) Traction-separation relation of the bond-mediated interface.

Besides the peeling velocity, Fig. 6(a) shows the strip profile under different levels of the peeling force. Clearly, the strip is peeled off more "sharply" and tends to have a smaller process zone under a larger peeling force. Because of the convertibility between time- and space-dependent quantities mentioned above, the separation history of individual bonds can be determined by the trajectory of the strip deflection in the process zone and peeling velocity. Specifically, the bond separation history and bond traction under different values of the peeling force are plotted in Figs. 6(b) and 6(c), respectively. It can be seen that bonds are separated in an exponential manner, with different rates that are related to the peeling velocity of the strip under different peeling force levels. Furthermore, 
bonds that are separated faster generate larger interfacial tractions. In contrast to the previous peel studies where the rate sensitivity originates from the bulk viscoelasticity of the materials (Chen et al., 2013), or from the plastic flow near the peeling front (Neggers et al., 2015), the rate dependence here stems from the mechano-kinetic evolution of molecular bonds at the interface.
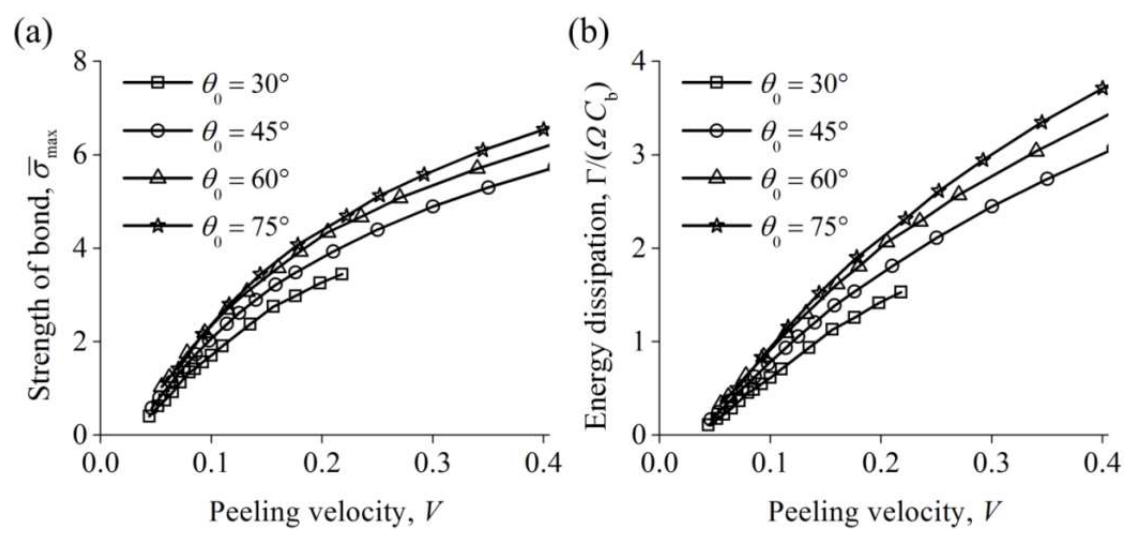

Fig. 7. (a) Rate-dependent interfacial strength and (b) energy dissipation as the peeling front propagates at different velocities.

The amount of energy dissipated during this process can be calculated by integrating the bond traction $\bar{\sigma}$ over the separation $W$ (Fig. 6(d)), which is

$$
\Gamma=\int_{W=0}^{W=+\infty} \bar{\sigma} \mathrm{d} W
$$

The dependence of the maximum bond traction $\bar{\sigma}_{\max }$ on the peeling velocity $V$ is shown in Fig. 7(a). Not surprisingly, the interface appears to be stronger under higher peeling rate. Similar to the maximum bond traction, the energy dissipation $\Gamma$ (normalized by the apparent adhesion energy $\Omega C_{\mathrm{b}}$ ) also increases with the peeling velocity (Fig. 7(b)). 

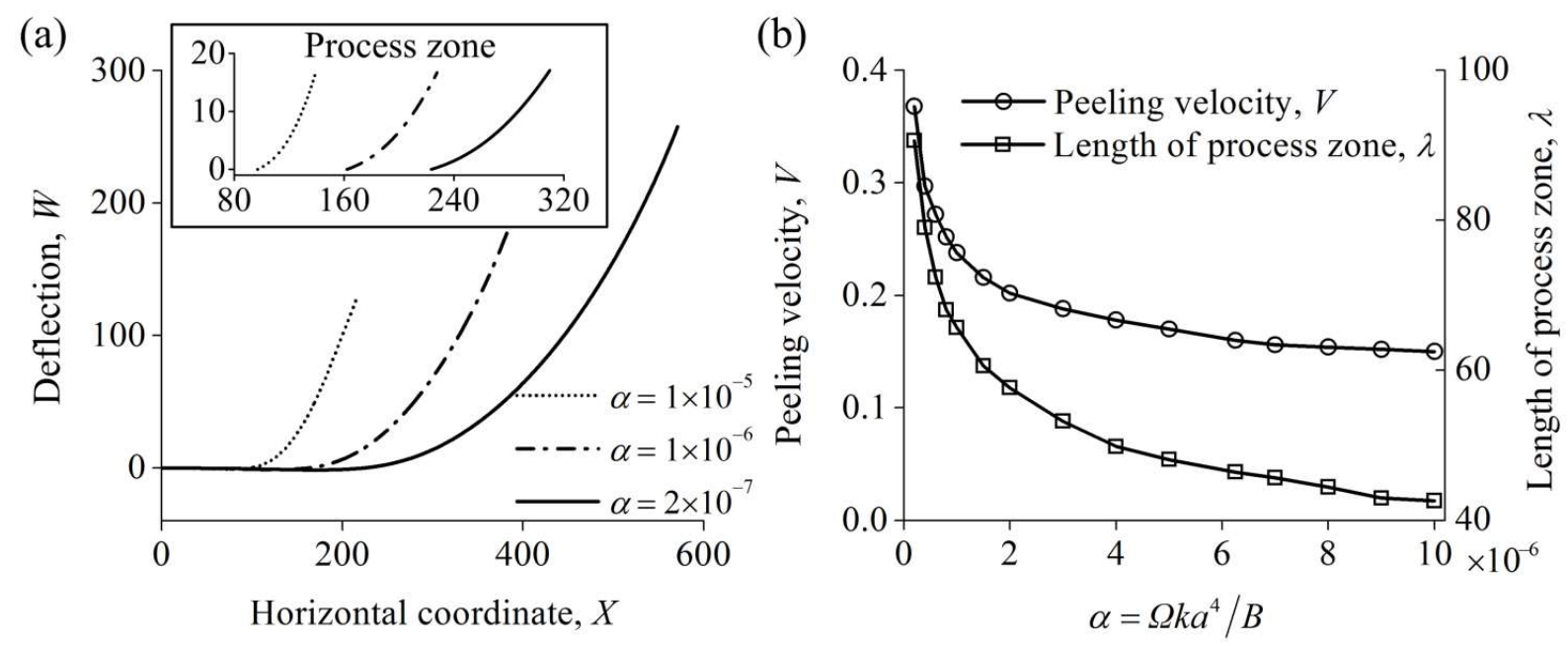

Fig. 8. (a) Deflection profile of the strip and (b) peeling velocity governed by the parameter $\alpha=\Omega \mathrm{ka}^{4} / B$.

Next, we examine the role of different parameters in determining the resultant peeling velocity. As discussed earlier, under steady-state peeling, the strip will maintain a stable profile when observed in the moving frame, as a result of the balance between bond traction and elastic stresses generated within the strip. According to Eqs. (15) and (16), the dimensionless parameter $\alpha$ $\left(=\Omega k a^{4} / B\right)$ is expected to play a key role here. The influence of $\alpha$ on the shape of the deformed strip is illustrated in Fig. 8(a). Note that the range of $\alpha$ examined here is between $10^{-7}$ and $10^{-5}$ given that $k$ is around $1-5 \mathrm{pN} / \mathrm{nm}$, the bond density $\Omega$ is of the order of $100-1000 \mu^{-2}$, and the bending modulus of the strip $B$ varies from 35 to $300 k_{\mathrm{B}} T_{\mathrm{m}}$. The role of $\alpha$ is especially evident in regulating the size of the peeling process zone where rupture of molecular bonds takes place. Figure $8(\mathrm{~b})$ shows that the length of the process zone decreases by half when $\alpha$ increases from $2 \times 10^{-7}$ to $10^{-5}$, suggesting that strips with smaller bending modulus or denser bonds will be peeled more sharply with a smaller process zone. Figure $8(\mathrm{~b})$ shows that the peeling velocity $V$ becomes smaller as $\alpha$ increases, indicating that the peeling process is slower at higher bond density, larger bond stiffness or lower bending rigidity of the strip. 

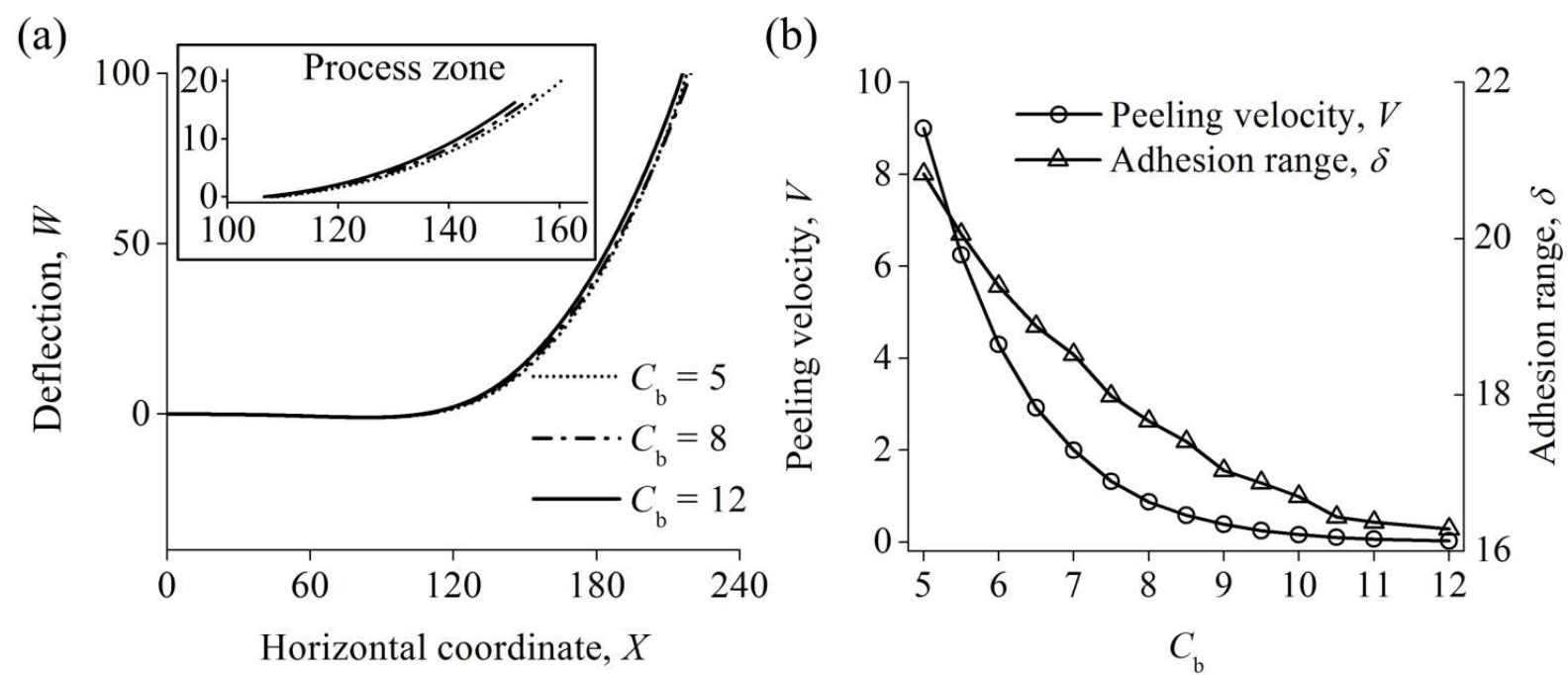

Fig. 9. Effects of the depth of bond energy well $C_{\mathrm{b}}$ on (a) deflection profile of the strip and (b) peeling velocity.

In addition to $\alpha$, the depth of bond energy well $C_{\mathrm{b}}$ also affects how peeling progresses. Interestingly, the deformed strip profile is found to be rather insensitive to the variations of $C_{\mathrm{b}}$ (Fig. 9(a)). However, the peeling velocity is significantly reduced with increasing $C_{b}$; see Fig. 9(b). The adhesive interaction range $\delta$ is also plotted against $C_{\mathrm{b}}$ in Fig. (9b).
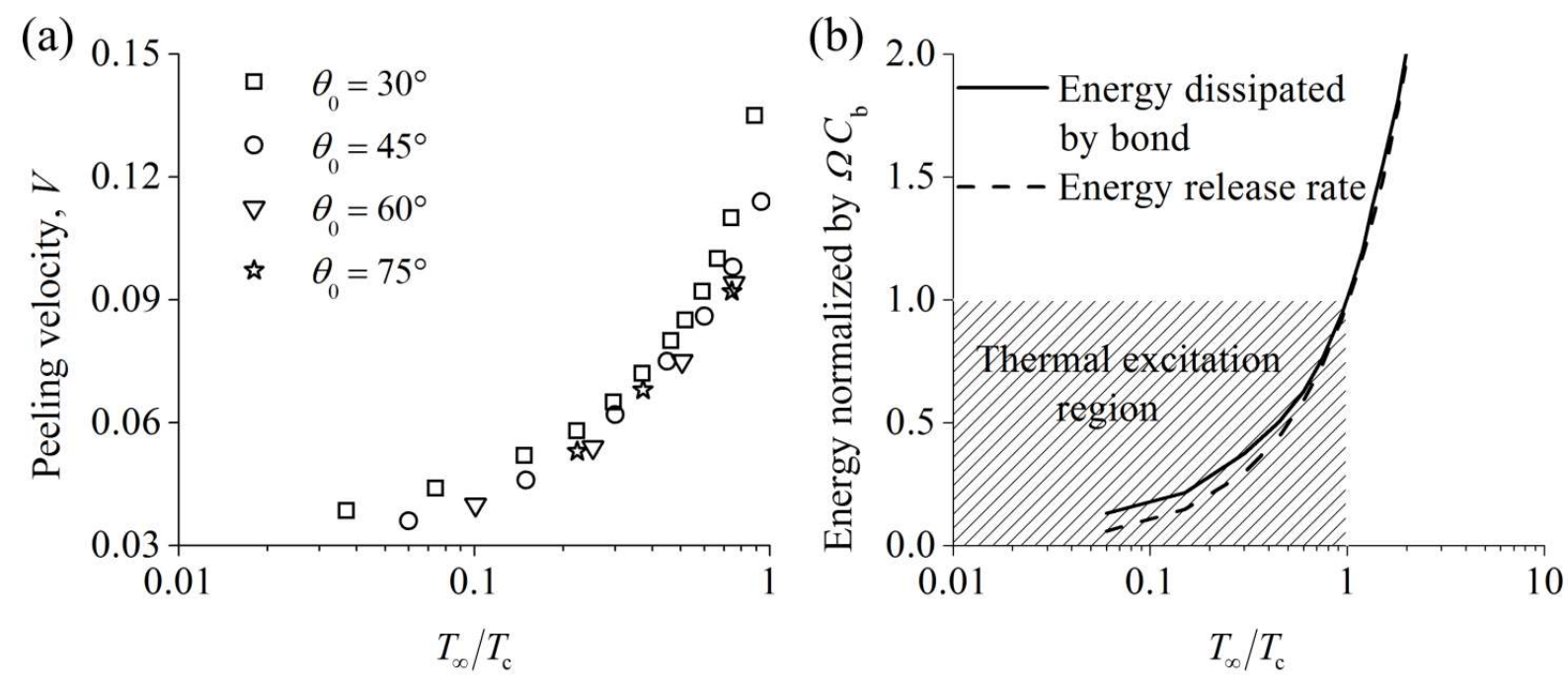

Fig. 10. (a) Peeling velocity and (b) energy dissipation at subcritical values of the peeling force.

It should be pointed out that, in most previous studies (Kendall, 1975; Williams, 1997; Begley et 
al., 2013), the criterion for the onset of peeling was obtained by equating the energy $G$ released per unit progression of peeling with the interfacial energy $W_{0}$, i.e. $G=W_{0}$. In the present setting, $W_{0}=\Omega C_{\mathrm{b}}$ and the energy release rate can be calculated as (Kendall, 1975)

$$
G=T_{\infty}\left(1-\cos \theta_{0}+\varepsilon / 2\right)
$$

where $\varepsilon$ is the tensile strain within the strip. Note that Eq. (29) reduces to the well-known Young equation if the tension term is neglected. Taking the thickness of the strip to be $h=20 \mathrm{~nm}$, a typical value of cytomembrane (Rand, 1964), the tensile strain can be calculated through $\varepsilon=T_{\infty} h^{2} /(24 B)$. Thus, the critical peeling force for peeling is identified as

$$
T_{\mathrm{c}}=\frac{2 \Omega C_{\mathrm{b}}}{\sqrt{\left(1-\cos \theta_{0}\right)^{2}+\Omega C_{\mathrm{b}} h^{2} / 6 B}+\left(1-\cos \theta_{0}\right)} .
$$

From this classical and deterministic point of view, peeling occurs only when the applied peeling force exceeds the critical value, i.e. $T>T_{\mathrm{c}}$. However, in the present study of peeling mediated by molecular bonds whose association and dissociation are strongly stochastic, peeling progresses irrespective of the value of $T$, even when $T / T_{\mathrm{c}}<1$, as demonstrated in Fig. 10(a). It is also interesting to observe that for $T / T_{\mathrm{c}}<1$, the energy dissipated by interfacial bonding is larger than the released elastic energy (Fig. 10(b)), implying that this process is assisted by thermal fluctuations.

\section{Conclusion}

In this work, we have revisited the classical problem of the peeling of an elastic strip from a substrate where the adhesion is mediated by molecular bonds at the interface. Upon the application of an inclined tensile peeling force, the strip experiences stretching, bending and tractions from the interfacial bonds that constantly undergo force- and fluctuation-assisted state evolution. We have shown that the space- and time-varying survival of these bonds largely determines how this peeling 
process progresses. Unlike classical and deterministic models where the peeling front propagates only when the applied peeling force is above a threshold level, the present statistical study has revealed the following behaviors:

(i) molecular bonds undergo kinetic rupture and rebinding with intrinsic time scales, resulting in a strongly rate-dependent peeling response;

(ii) the peeling velocity is heavily influenced by the association/dissociation kinetics of the interfacial bonds, and various factors such as bond density, bond stiffness and bending modulus of the strip through a dimensionless parameter $\alpha=\Omega k a^{4} / B$;

(iii) most importantly, because of the probabilistic nature of our model, peeling occurs even when the applied peeling force is below the critical value predicted by deterministic models.

The energy dissipation of molecular bonds is shown to be strongly rate-dependent, rooted in the diffusive evolution of bond state at the interface. We believe that the present work may be useful in understanding the mechano-chemical coupling in receptor-ligand mediated adhesion, where convoluted kinetic processes are regulated by non-uniform and time-varying elastic fields through traction and displacement. This study can also be extended to model the locomotion of cells, where the trailing membrane is constantly peeled away from substrates by breaking molecular bonds.

\section{Acknowledgment}

This work was supported by the National Natural Science Foundation of China (Nos. 11621062 , 11672268, 11402193 and 11572273), and Zhejiang Provincial Natural Science Foundation of China (No. LR16A020001). Y.L. also gratefully acknowledges support from the Research Grants Council (Project No. HKU 7147/13E, HKU 17205114 and HKU 17211215) of the Hong Kong Special 
Administration Region and from the University of Hong Kong (Project No. 201411159167 and 201511159165). H.G. acknowledges support from the National Science Foundation under Grant CMMI-1562904. 


\section{References}

1. Alberts, B., Johnson, A., Lewis, J., Raff, M., Roberts, K., Walter, P., 2002. Molecular Biology of the Cell, 4th ed. Garland Science, New York.

2. Arnold, M., Cavalcanti-Adam, E.A., Glass, R., Blummel, J., Eck, W., Kantlehner, M., Kessler, H., Spatz, J.P., 2004. Activation of integrin function by nanopatterned adhesive interfaces. ChemPhysChem 5, 383-388.

3. Begley, M.R., Collino, R.R., Israelachvili, J.N., McMeeking, R.M., 2013. Peeling of a tape with large deformations and frictional sliding. Journal of the Mechanics and Physics of Solids 61, $1265-1279$.

4. Bell, G.I., 1978. Models for specific adhesion of cells to cells. Science 200, 618-627.

5. Boal, D., 2002. Mechanics of the Cell. Cambridge University Press.

6. Chen, B., Wu, P., Gao, H., 2009. Pre-tension generates strongly reversible adhesion of a spatula pad on substrate. Journal of the Royal Society Interface 6, 529-537.

7. Chen, H., Feng, X., Huang, Y., Huang, Y.G., Rogers, J.A., 2013. Experiments and viscoelastic analysis of peel test with patterned strips for applications to transfer printing. Journal of the Mechanics and Physics of Solids 61, 1737-1752.

8. Chen, W., Lou, J.Z., Evans, E.A., Zhu, C., 2012. Observing force-regulated conformational changes and ligand dissociation from a single integrin on cells. Journal of Cell Biology 199, 497-512.

9. Cheng, Q.H., Chen, B., Gao, H.J., Zhang, Y.W., 2012. Sliding-induced non-uniform pretension governs robust and reversible adhesion: a revisit of adhesion mechanisms of geckos. Journal of the Royal Society Interface 9, 283-291. 
10. Dembo, M., Torney, D.C., Saxman, K., Hammer, D., 1988. The reaction-limited kinetics of membrane-to-surface adhesion and detachment. Proceedings of the Royal Society Series B-Biological Sciences 234, 55-83.

11. Erdmann, T., Schwarz, U.S., 2004a. Stability of adhesion clusters under constant force. Physical Review Letters 92, 108102.

12. Erdmann, T., Schwarz, U.S., 2004b. Stochastic dynamics of adhesion clusters under shared constant force and with rebinding. Journal of Chemical Physics 121, 8997-9017.

13. Evans, E.A., 1985a. Detailed mechanics of membrane-membrane adhesion and separation. 1. Continuum of molecular cross-bridges. Biophysical Journal 48, 175-183.

14. Evans, E.A., 1985b. Detailed mechanics of membrane-membrane adhesion and separation. 2. Discrete kinetically trapped molecular cross-bridges. Biophysical Journal 48, 185-192.

15. Evans, E., Ritchie, K., 1997. Dynamic strength of molecular adhesion bonds. Biophysical Journal $72,1541-1555$.

16. Freund, L.B., 2009a. Characterizing the resistance generated by a molecular bond as it is forcibly separated. Proceedings of the National Academy of Sciences of the United States of America 106, $8818-8823$.

17. Freund, L.B., 2009b. The stiffness of a biomembrane force probe vesicle. Mathematics and Mechanics of Solids 14, 148-160.

18. Freund, L.B., 2014. Brittle crack growth modeled as the forced separation of chemical bonds within a K-field. Journal of the Mechanics and Physics of Solids 64, 212-222.

19. Freund, L.B., Lin, Y., 2004. The role of binder mobility in spontaneous adhesive contact and implications for cell adhesion. Journal of the Mechanics and Physics of Solids 52, 2455-2472. 
20. Gao, H., Qian, J., Chen, B., 2011. Probing mechanical principles of focal contacts in cell-matrix adhesion with a coupled stochastic-elastic modelling framework. Journal of the Royal Society Interface $8,1217-1232$.

21. Gialamas, P., Voelker, B., Collino, R.R., Begley, M.R., McMeeking, R.M., 2014. Peeling of an elastic membrane tape adhered to a substrate by a uniform cohesive traction. International Journal of Solids and Structures 51, 3003-3011.

22. Jiang, H.Y., Qian, J., Lin, Y., Ni, Y., He, L.H., 2015. Aggregation dynamics of molecular bonds between compliant materials. Soft Matter 11, 2812-2820.

23. Jin, F., Guo, X., 2013. Mechanics of axisymmetric adhesive contact of rough surfaces involving power-law graded materials. International Journal of Solids and Structures 50, 3375-3386.

24. Ju, L.N., Qian, J., Zhu, C., 2015. Transport regulation of two-dimensional receptor-ligand association. Biophysical Journal 108, 1773-1784.

25. Kendall, K., 1971. Adhesion and surface energy of elastic solids. Journal of Physics D-Applied Physics 4, 1186-1195.

26. Kendall, K., 1975. Thin-film peeling - elastic term. Journal of Physics D-Applied Physics 8, $1449-1452$.

27. Kramers, H. A, 1940. Brownian motion in the field of force and the diffusion model of chemical reactions. Physica 7, 284-304.

28. Kumar, S., Weaver, V.M., 2009. Mechanics, malignancy, and metastasis: the force journey of a tumor cell. Cancer and Metastasis Reviews 28, 113-127.

29. Leckband, D., Israelachvili, J., 2001. Intermolecular forces in biology. Quarterly Reviews of Biophysics 34, 105-267. 
30. Lin, J., Lin, Y., Qian, J., 2014. Statistical pull off of nanoparticles adhering to compliant substrates. Langmuir 30, 6089-6094.

31. Lin, Y., Yao, S., Xu, Q., 2010. Dynamic adhesion energy between surfaces connected by molecular bonds and its application to peel test. Cellular and Molecular Bioengineering 3, 247-255.

32. Lu, Z.X., Yu, S.W., Wang, X.Y., Feng, X.Q., 2007. Effect of interfacial slippage in peel test: Theoretical model. European Physical Journal E 23, 67-76.

33. Merkel, R., Nassoy, P., Leung, A., Ritchie, K., Evans, E., 1999. Energy landscapes of receptor-ligand bonds explored with dynamic force spectroscopy. Nature 397, 50-53.

34. Molinari, A., Ravichandran, G., 2008. Peeling of elastic tapes: effects of large deformations, pre-straining, and of a peel-zone model. Journal of Adhesion 84, 961-995.

35. Neggers, J., Hoefnagels, J.P.M., van der Sluis, O., Geers, M.G.D., 2015. Multi-scale experimental analysis of rate dependent metal-elastomer interface mechanics. Journal of the Mechanics and Physics of Solids 80, 26-36.

36. Peng, Z.L., Chen, S.H., 2012. Effect of pre-tension on the peeling behavior of a bio-inspired nano-film and a hierarchical adhesive structure. Applied Physics Letters 101, 163702.

37. Peng, Z.L., Chen, S.H., 2015. Peeling behavior of a thin-film on a corrugated surface. International Journal of Solids and Structures 60-61, 60-65.

38. Pesika, N.S., Tian, Y., Zhao, B., Rosenberg, K., Zeng, H., McGuiggan, P., Autumn, K., Israelachvili, J.N., 2007. Peel-zone model of tape peeling based on the gecko adhesive system. Journal of Adhesion 83, 383-401.

39. Qian, J., Lin, Y., Jiang, H.Y., Yao, H.M., 2013. Bond formation of surface-tethered 
receptor-ligand pairs in relative separation. Applied Physics Letters 103, 223702.

40. Qian, J., Wang, J.Z., Gao, H., 2008. Lifetime and strength of adhesive molecular bond clusters between elastic media. Langmuir 24, 1262-1270.

41. Qian, J., Wang, J.Z., Lin, Y., Gao, H., 2009. Lifetime and strength of periodic bond clusters between elastic media under inclined loading. Biophysical Journal 97, 2438-2445.

42. Rand, R.P., 1964. Mechanical properties of the red cell membrane. II. Viscoelastic breakdown of the membrane. Biophysical Journal 4, 303-316.

43. Rief, M., Gautel, M., Oesterhelt, F., Fernandez, J.M., Gaub, H.E., 1997. Reversible unfolding of individual titin immunoglobulin domains by AFM. Science 276, 1109-1112.

44. Roca-Cusachs, P., Iskratsch, T., Sheetz, M.P., 2012. Finding the weakest link - exploring integrin-mediated mechanical molecular pathways. Journal of Cell Science 125, 3025-3038.

45. Vossen, B.G., Schreurs, P.J.G., van der Sluis, O., Geers, M.G.D., 2014. Multi-scale modeling of delamination through fibrillation. Journal of the Mechanics and Physics of Solids 66, 117-132.

46. Wei, Y.J., 2014. A stochastic description on the traction-separation law of an interface with non-covalent bonding. Journal of the Mechanics and Physics of Solids 70, 227-241.

47. Williams, J.G., 1997. Energy release rates for the peeling of flexible membranes and the analysis of blister tests. International Journal of Fracture 87, 265-288.

48. Xia, S.M., Ponson, L., Ravichandran, G., Bhattacharya, K., 2013. Adhesion of heterogeneous thin films I: elastic heterogeneity. Journal of the Mechanics and Physics of Solids 61, 838-851.

49. Xia, S.M., Ponson, L., Ravichandran, G., Bhattacharya, K., 2015. Adhesion of heterogeneous thin films II: adhesive heterogeneity. Journal of the Mechanics and Physics of Solids 83, 88-103.

50. Xu, G.K., Yang, C., Du, J., Feng, X.Q., 2014. Integrin activation and internalization mediated by 
extracellular matrix elasticity: a biomechanical model. Journal of Biomechanics 47, 1479-1484.

51. Zhang, W., Jin, F., Zhang, S.L., Guo, X., 2014. Adhesive contact on randomly rough surfaces based on the double-Hertz model. Journal of Applied Mechanics 81, 051008. 